\title{
Wave propagation of spectral energy content in a granular chain
}

\author{
Rohit Kumar Shrivastava ${ }^{1, \star}$ and Stefan Luding ${ }^{1, \star \star}$ \\ ${ }^{1}$ Multiscale Mechanics (MSM), MESA+, Faculty of Engineering Technology (CTW), PO Box 217, 7500 AE Enschede, Netherlands.
}

\begin{abstract}
A mechanical wave is propagation of vibration with transfer of energy and momentum. Understanding the spectral energy characteristics of a propagating wave through disordered granular media can assist in understanding the overall properties of wave propagation through inhomogeneous materials like soil. The study of these properties is aimed at modeling wave propagation for oil, mineral or gas exploration (seismic prospecting) or non-destructive testing of the internal structure of solids. The focus is on the total energy content of a pulse propagating through an idealized one-dimensional discrete particle system like a mass disordered granular chain, which allows understanding the energy attenuation due to disorder since it isolates the longitudinal P-wave from shear or rotational modes. It is observed from the signal that stronger disorder leads to faster attenuation of the signal. An ordered granular chain exhibits ballistic propagation of energy whereas, a disordered granular chain exhibits more diffusive like propagation, which eventually becomes localized at long time periods. For obtaining mean-field macroscopic/continuum properties, ensemble averaging has been used, however, such an ensemble averaged spectral energy response does not resolve multiple scattering, leading to loss of information, indicating the need for a different framework for micro-macro averaging.
\end{abstract}

\section{Introduction}

Disorder in granular materials (like soil) can manifest in many ways (contact disorder, geometrical disorder, asphericity, layering, etc.) from grain level to system level. All may have an effect on the mechanical wave transmission through the granular material in it's own unique way (for instance, contact disorder due to tiny polydispersity can reduce the mechanical wave speed [9]). Knowing this effect can aid us in numerous ways for subsurface exploration or for non-destructive testing. Thus, there is a need to study the effects of disorder individually and hence the focus in this article will only be on mass disorder, for which 1D granular chain has been chosen so that the P-wave mode is isolated from shear or rotational modes. A mechanical wave propagating through this $1 \mathrm{D}$ granular chain is bound to suffer from multiple scattering. However, regardless of scattering, linear waves preserve some coherence and that manifests as intensity correlations ([8]). The results obtained from the chain also represent attributes of both longitudinal P-waves (compressional) and S-waves (shear) in a 3D system as stated in [10]; The frequency filtering effects are similar to those in a 3D system, as observed in [9]. All the more reason to study energy content and spectral energy response of the propagating wave.

\footnotetext{
^e-mail: r.k.shrivastava@utwente.nl

${ }^{\star}$ e-mail: s.luding@utwente.nl
}

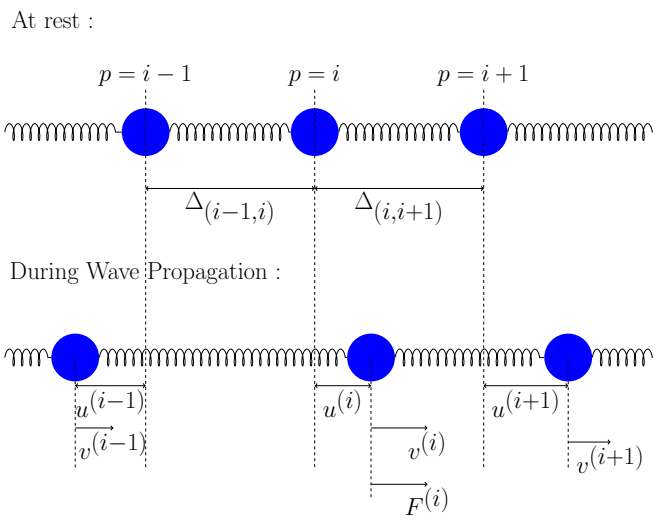

Figure 1. Chain of granular elements during dynamic wave propagation with length scaled by the characteristic equilibrium distance $\tilde{\Delta_{o}}$.

\section{Micromechanical model of $1 \mathrm{~d}$ chain}

A one dimensional granular chain of mass disordered mesoscopic particles is modeled using only a repulsive interaction force,

$$
F_{(i, i+1)}=\kappa_{(i, i+1)} \delta_{(i, i+1)}=\kappa_{(i, i+1)}\left(\Delta_{(i, i+1)}-\left(u^{(i)}-u^{(i+1)}\right)\right)
$$

The granular chain has a high pre-confining force which does not allow opening and closing of contacts (no occurrence of Nesterenko's sonic vacuum $[2,11])$. Hence, a linear contact model is valid to compute the repulsive 
Table 1. Dimensional, scaling and hence, the non-dimensionalized parameters.

\begin{tabular}{lll}
\hline Dimensional & Scaling & Non-dimensional \\
\hline$\tilde{m}^{(i)}$ & $\tilde{m}_{o}$ & $b^{(i)}=\tilde{m}^{(i)} / \tilde{m}_{o}$ \\
$\tilde{\kappa}_{(i, j)}$ & $\tilde{\kappa}_{o}$ & $\kappa_{(i, j)}=\tilde{\kappa}_{(i, j)} / \tilde{\kappa}_{o}$ \\
$\tilde{\Delta}_{(i, j)}$ & $\tilde{\Delta}_{o}$ & $\Delta_{(i, j)}=\tilde{\Delta}_{(i, j)} / \tilde{\Delta}_{o}$ \\
\hline
\end{tabular}

interaction forces from the overlap $\left(\delta_{(i, i+1)}\right)$ [4] for small amplitudes. Table 1 contains the scaling parameters required to arrive at the non-dimensional equation of motion of particle $i . b^{(i)}$ is the non-dimensional mass of particle $i$ and $\tilde{m}_{o}$ is the average mass of the particles. $\kappa_{(i, j)}$ and $\Delta_{(i, j)}$ are the non-dimensionalized stiffness and overlap between particles $i$ and $j$, respectively, scaled by the characteristic stiffness $\left(\tilde{\kappa}_{o}\right)$ and the corresponding characteristic overlap $\left(\tilde{\Delta_{o}}\right)$. Time $(t)$ is scaled by $\sqrt{\frac{\tilde{m}_{o}}{\tilde{\kappa}_{o}}}$ (natural frequency of the chain) to give non-dimensional time $(\tau)$. The equation of motion of a general particle $i$ is given by,

$$
\begin{aligned}
F^{(i)}(\tau)=b^{(i)} \frac{\mathrm{d}^{2} u^{(i)}}{\mathrm{d} \tau^{2}} & =\kappa_{(i-1, i)}\left[\Delta_{(i-1, i)}-\left(u^{(i)}-u^{(i-1)}\right)\right] \\
& -\kappa_{(i, i+1)}\left[\Delta_{(i, i+1)}-\left(u^{(i+1)}-u^{(i)}\right)\right] \\
& =-\left[2 u^{(i)}-u^{(i-1)}-u^{(i+1)}\right] .
\end{aligned}
$$

where $u^{(i)}$ is the non-dimensionalized displacement of particle $i$, scaled by characteristic overlap $\left(\tilde{\Delta}_{o}\right)$. Since, the focus of the analyses here has been only on the effects of mass disorder, it makes us take all interparticle stiffnesses equal to the characteristic stiffness $\left(\tilde{\kappa}_{o}\right)$ which makes the non-dimensionalized interparticle stiffness, $\kappa_{(i, j)}=1$ and implicitly the interparticle overlap at rest becomes, $\Delta_{(i, j)}=$ 1. Using $\kappa_{(i, j)}=1, \Delta_{(i, j)}=1$ and assembling equations (2) in the granular chain, we arrive at a matrix form of these equations given by,

$$
\mathbf{M} \frac{\mathrm{d}^{2} \mathbf{u}}{\mathrm{d} \tau^{2}}=\mathbf{K u},
$$

where $\mathbf{M}$ is a diagonal matrix containing $b^{(1)}, b^{(2)}, \ldots . b^{(N)}$ (masses of the particles) along the diagonal. $\mathbf{K}$ contains -2 along the diagonal, +1 along sub-diagonal and superdiagonal elements and 0 elsewhere. Defining $\mathbf{A}=\mathbf{M}^{-1} \mathbf{K}$ and assuming normal mode motion of particles, equation (3) becomes $\mathbf{A u}=\omega^{2} \mathbf{u}$ with $\omega_{(j)}^{2}$ as eigenvalues of the matrix $\mathbf{A}$ and $\omega_{(j)}$ as eigenfrequencies. Defining $(N \times N)$ matrix $\mathbf{S}$, containing eigenvectors of $\mathbf{A}, \mathbf{s}_{(j)}$ as the columns and arranged in increasing order with respect to their corresponding eigenfrequencies. $\mathbf{S}$ is further orthonormalized to satisfy the relation $\mathbf{S}^{\mathbf{T}} \mathbf{M S}=\mathbf{I}$ (Identity matrix). Using initial conditions $\mathbf{v}_{o}=\left[\begin{array}{lllll}v_{0} & 0 & 0 & \ldots & 0\end{array}\right]$ (initial velocity) and $\mathbf{u}_{o}=0$ (initial displacement) as impulse boundary conditions, we get the displacement and velocity history of particles as,

$$
\begin{aligned}
& u^{(p)}(\tau)=v_{o} \sum_{j=1}^{N} \frac{S_{p j} S_{1 j} \sin \left(\omega_{(j)} \tau\right)}{\omega_{(j)}} \\
& v^{(p)}(\tau)=v_{o} \sum_{j=1}^{N} S_{p j} S_{1 j} \cos \left(\omega_{(j)} \tau\right)
\end{aligned}
$$

\subsection{Mass disorder and disorder parameter}

The diagonal elements of the mass matrix $\mathbf{M}, b^{(1)}, b^{(2)}$, .... $b^{(N)}$ (masses of the particles) have been selected from a normal distribution $f^{(n)}(b)=\frac{1}{\xi \sqrt{2 \pi}} e^{-\frac{(b-1)^{2}}{2 \xi^{2}}}$ whose standard deviation $(\xi)$ quantifies the disorder of the granular chain. The scaled average of the distribution is 1 . The aforementioned model has been used previously in [10] for a frequency filtering study of the displacement wave (mass disorder dependent frequency filtering and attenuation) and to show that the shape of disorder probability (binary, uniform, normal or any other distribution) produce quantitatively similar frequency filtering, up to a certain strength of disorder.

\subsection{Total energy signal from displacement and velocity histories}

We go one step further and determine the total energy signal per particle which is given by,

$$
E^{(p)}(\tau)=\underbrace{\frac{1}{2} b^{(p)}\left(v^{(p)}(\tau)\right)^{2}}_{\text {Kinetic Energy }}-\underbrace{\frac{1}{2} F^{(p)}(\tau)\left(u^{(p)}(\tau)\right)}_{\text {Potential Energy }} .
$$

The position of the center of total energy in the granular chain is given by [5],

$$
R(\tau)=\frac{1}{E_{\text {tot }}} \sum_{p=1}^{N} p E^{(p)}(\tau), \text { with, } E_{t o t}=\sum_{p=1}^{N} E^{(p)}(\tau)
$$

The squared width of the propagating pulse is,

$$
r^{2}(\tau)=\frac{1}{E_{\text {tot }}} \sum_{p=1}^{N}(p-R(\tau))^{2} E^{(p)}(\tau)
$$

\subsection{Model system and $\langle$ ensemble $\rangle$ averaging}

A 256 element long granular chain has been used with disorder parameters $\xi=0.0, \xi=0.1, \xi=0.2, \xi=0.35$, $\xi=0.5$ and $\xi=0.8$ for the mass distribution of the chain. The time duration for the computation was 256 (time duration till which the pulse has propagated to about the last particle in the granular chain) and the time step utilized for the data output was 0.032. 500 ensembles/realizations of granular chains have been utilized and displacement, velocity, force and hence, total energy histories were obtained and ensemble averaged for different disorder parameters. From here onwards angular brackets $(\langle\rangle)$ will denote ensemble averaging.

\section{Results}

$\langle R(\tau)\rangle,\left\langle r^{2}(\tau)\right\rangle,\left\langle E^{(p)}(\tau)\right\rangle$ and the Fourier transform of $\left\langle E^{(p)}(\tau)\right\rangle\left(\left\langle\mathcal{E}^{(p)}(\omega)\right\rangle\right)$ are discussed in this Section. 


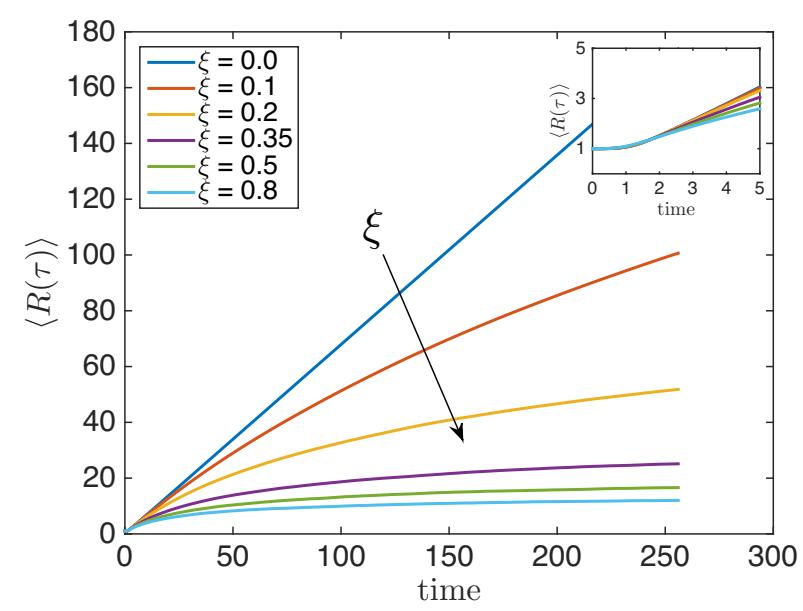

Figure 2. Propagation of center of energy $\langle R(\tau)\rangle$ in different disordered granular chains with the arrow indicating increasing disorder.

\subsection{Propagation of center of energy}

In order to monitor the propagation of the center of energy during propagation of a pulse in a disordered granular chain. $R(\tau)$ from equation (6) has been computed. In Figure 2, the center of energy of propagating pulses for 500 realizations is plotted against time for different disorders. Initially the center of energy does not propagate (as shown in the inset figure), this is the time during which the initial high frequency impulse is self-demodulated [6] by the granular chain (in contrast to a Gaussian pulse [5]) and after this short time interval, for a short time, the center of energy propagates with the same speed for different disorder parameters. $\xi=0.0$ has linear (ballistic) propagation of center of energy whereas, $\xi>0$ displays nonlinear propagation of the center of energy with propagation speed decreasing with increasing time. Stronger disorders have stronger decrease in propagation speed with increase in time. Unlike $\xi=0.0$, higher $\xi$ have the center of energy becoming confined in a finite space and this confinement space is smaller for stronger disorder. Such confinement of energy of mechanical waves has often been attributed to Anderson's localization [5], when elastic waves get trapped in a confined space due to the disorder in the medium [1] which also becomes more enhanced with increase in disorder.

\subsection{Squared width of propagating pulse}

The ensemble averaged, squared width of the pulse obtained from equation (7) has been plotted in Figure 3 against time in log-log scale to see whether the energy dispersion is diffusive in nature. It is observed that for an impulse response, after the self-demodulation time interval (recognized from the inset in Figure 2), the energy propagation is slightly superballistic for low disorder parameters (eg. $\xi=0.1$ ) and gets nonlinear towards diffusive for high disorder parameters (eg. $\xi=0.8$ ).

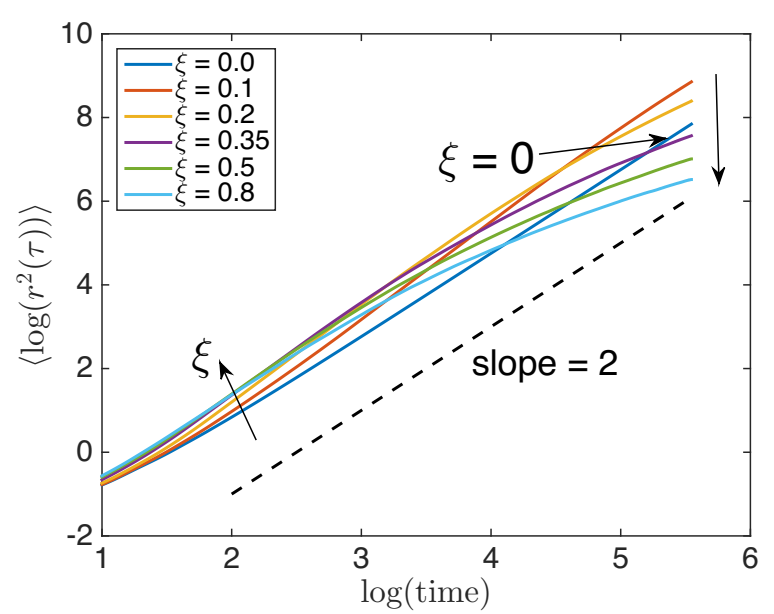

Figure 3. Squared width of the pulse with respect to time (after self-demodulation) for different parameters in log-log scale. Line is a guide to the eye. Arrows indicates the trend for $\xi>0$.

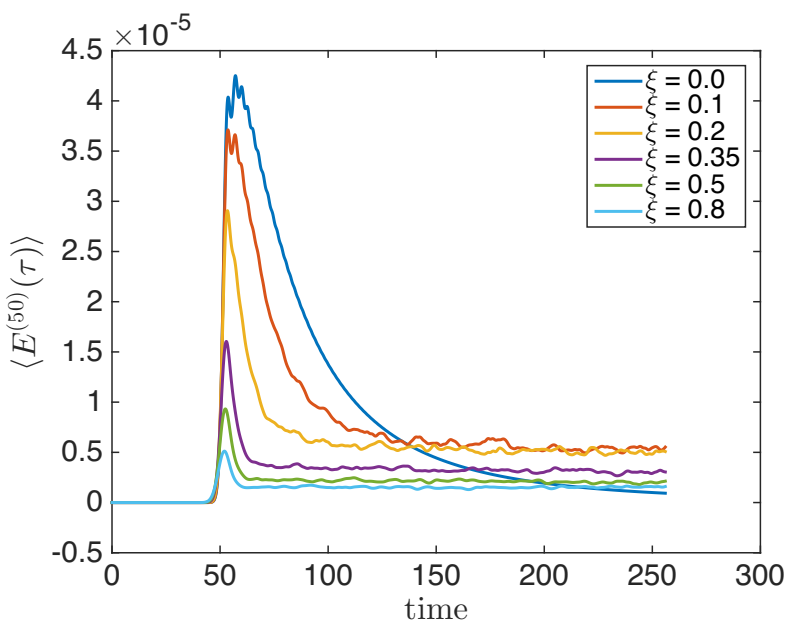

Figure 4. Averaged Energy history of $50^{\text {th }}$ particle in a 256 particles long granular chain for different disorder parameters.

\subsection{Spectral energy analysis}

The ensemble averaged total energy signal $\left(E^{(p)}(\tau)\right)$ for the $50^{\text {th }}$ particle in a 256 long chain for different disorder parameters is plotted in Figure 4. We can observe that the initial coherent wavefront is attenuating faster in stronger disordered media as indicated in [10], the peak of this coherent wavefront gives the maximum peak energy $\left(E_{P}\right)$ attained by the particle during wave propagation which has been plotted in Figure 5(a) for different disorder parameters $(\xi)$. Due to ensemble averaging, the multiply scattered incoherent part [7] has attained a constant value (with very minor fluctuations) giving the residual energy $\left(E_{R}\right)$ contained in the particle after the initial pulse has passed across the particle, this residual energy at $\tau=256$ has also been plotted in Figure 5(a) for different $\xi$, it shows that the residual energy contained in the particle decreases with increasing disorder. The ratio between the residual energy and the peak energy shows an increasing trend as shown 

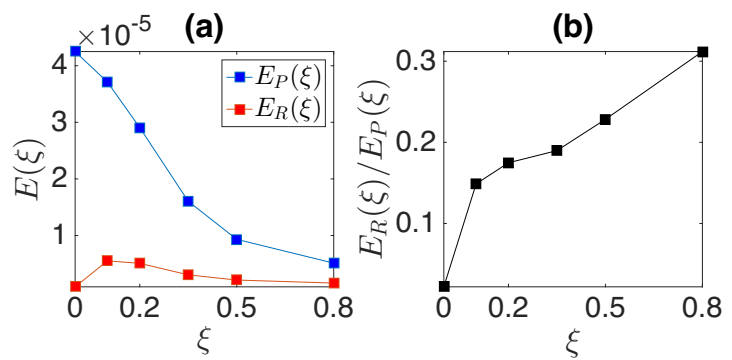

Figure 5. (a) Peak energy \& Residual energy and (b) their ratio at the $50^{\text {th }}$ particle for different $\xi$.

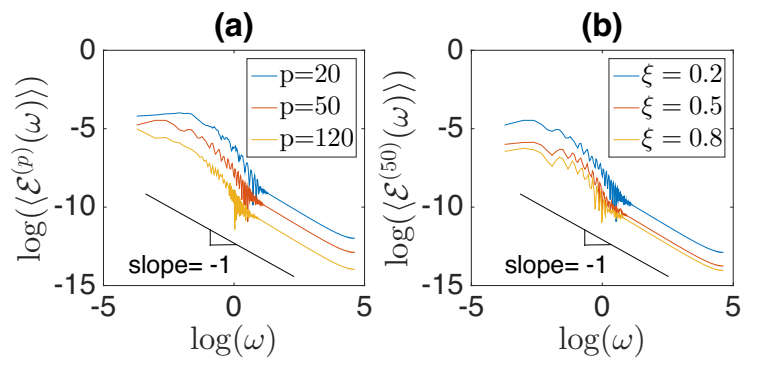

Figure 6. Ensemble averaged spectral energy response of (a) $20^{\text {th }}, 50^{\text {th }}$ and $120^{\text {th }}$ particle for $\xi=0.2$ and (b) $\xi=0.1,0.2$ and 0.5 for $50^{\text {th }}$ particle in log-log scale. The FFT-time window is 0 to 256 time units and time step is $\Delta t=0.0313$.

in 5(b). However, the spectral energy response for any particular particle remains qualitatively the same (Figure 6 (b)) irrespective of disorder, exhibiting only decrease in amplitude of the Fourier spectra and a slight shift towards lower frequency, resulting in loss of information contained in the multiple scattering part.

This suggests adoption of an alternative approach/model for the micro-macro transition rather than relying only on ensemble averaging. Distance and disorder (Figure 6(a) and Figure 6(b)) have no effect on this power law relationship, only the amplitude decreases with the position of the particle and disorder of the granular chain. This $\omega^{-1}$ power-law observation is not new as a similar relationship was observed with in Ref. [3].

\section{Conclusion}

It was observed in Section 3.1 that an ordered granular chain system has ballistic wave propagation of center of energy and a disordered system has a diffusive like propagation at larger times, when the energy becomes local- ized in space. For stronger disorder, this effect is enhanced. Figures 4 and 5 confirm, as mentioned in [10], that stronger disorder causes faster attenuation of signals. The spectral energy response exhibits a power-law relationship (Figure 6). A main cause of concern is that ensemble averaging causes the spectral energy response $\left(\mathcal{E}^{(p)}(\omega)\right)$ to become qualitatively similar for a particular particle irrespective of disorder. This results from the fact that averaging causes the multiply scattered part of the signal to cancel itself as it is not self-averaging hence, leading to loss of information. Therefore, a different micro-macro transition procedure needs to be developed.

\section{Acknowledgements}

This work is part of the Industrial Partnership Programme (IPP) 'Computational sciences for energy research' of the Foundation for Fundamental Research on Matter (FOM), which is part of the Netherlands Organisation for Scientific Research (NWO). This research programme is co-financed by Shell Global Solutions International B.V.

\section{References}

[1] P. W. Anderson, Phys. Rev. E 109, 1492-1505 (1958).

[2] V. F. Nesterenko, J. Appl. Mech. Tech. Phys. 24, 733743 (1983).

[3] Chu-heng Liu and R. Sidney Nagel, Phys. Rev. Lett. 68, 2301-2304 (1992).

[4] R. S. Sinkovits and S. Sen, Phys. Rev. E 54, $6857-$ 6865 (1996).

[5] P. B. Allen and J. Kelner, American Journal of Physics 66, 497-506 (1998).

[6] V. Tournat, V. E. Gusev and Castagnède, Phys. Rev. E 70, 056603 (2004)

[7] X. Jia, Phys. Rev. Lett. 93, 154303 (2004)

[8] R. L. Weaver, Science 307, 1568-1569 (2005)

[9] O. Mouraille and S. Luding, Ultrasonics 48, 498-505 (2008)

[10] B. P. Lawney and S. Luding, Acta Mechanica 225, 2385-2407 (2014).

[11] N. Upadhyaya, L.R. Gómez and V. Vitelli, Phys. Rev. X 4, 011045 (2014)

[12] J. O’Donovan, E. Ibraim, C. O’Sullivan, S. Hamlin, D. Muir Wood, G. Marketos, Granular Matter 18, 56 (2016)

[13] R. K. Shrivastava and S. Luding, Nonlin. Processes Geophys., submitted (2017). 